\title{
Haltere morphology and campaniform sensilla arrangement across Diptera
}

Sweta Agrawal ${ }^{\mathrm{a}}$, David Grimaldi ${ }^{\mathrm{b}}$, and Jessica L. Fox ${ }^{\mathrm{c} *}$

${ }^{\mathrm{a}}$ Department of Physiology and Biophysics, University of Washington, Seattle, WA, 98195, USA; sagrawal@u.washington.edu

${ }^{\mathrm{b}}$ Division of Invertebrate Zoology, American Museum of Natural History, New York, NY, 10024-5192; grimaldi@amnh.org

cDepartment of Biology, Case Western Reserve University, Cleveland, OH, 44106-7080; jlf88@case.edu

*Corresponding author:jlf88@case.edu 


\begin{abstract}
One of the primary specializations of true flies (order Diptera) is the modification of the hind wings into club-shaped halteres. Halteres are complex mechanosensory structures that provide sensory feedback essential for stable flight control via an array of campaniform sensilla at the haltere base. The morphology of these sensilla has previously been described in a small number of dipteran species, but little is known about how they vary across fly taxa. Using a synoptic set of specimens representing 42 families from all of the major infraorders of Diptera, we used scanning electron microscopy to map the gross and fine structures of halteres, including sensilla shape and arrangement. We found that several features of haltere morphology correspond with dipteran phylogeny: Schizophora generally have smaller halteres with stereotyped and highly organized sensilla compared to nematoceran flies. We also found a previously undocumented high variation of haltere sensilla shape in nematoceran dipterans, as well as the absence of a dorsal sensilla field in multiple families. Overall, variation in haltere sensilla morphology across the dipteran phylogeny provides insight into the evolution of a highly specialized proprioceptive organ and a basis for future studies on haltere sensory function.
\end{abstract}

Keywords: Diptera, halteres, sensilla, Hicks papillae, mechanoreceptors, campaniform sensilla

Abbreviations:

SEM, scanning electron micrograph; dSP, dorsal scapal plate; dBP, dorsal basal plate; dHP, dorsal Hicks papillae; dFS, dorsal flanking sensilla/sensillum; vHP, ventral Hicks papillae; vSP, ventral scapal plate

\title{
1. Introduction
}

Dipteran insects, the "true flies," include species that are amongst the most maneuverable of all flying animals. Much of this aerial agility is due to the modification of their hind wings into small, club-shaped mechanosensory organs that are entirely unattached to the forewings. These structures, the halteres, are a major defining characteristic of the order Diptera ("two wings"). Although the halteres do not generate lift, they experience multiple inertial forces during flight, and a sophisticated array of mechanosensory cells at the haltere base detects the cuticular deformations caused by these forces (Keil, 1997; Pringle, 1948). This sensory information is sent to motor neurons that steer the wings (Chan and Dickinson, 1996; Fayyazuddin and Dickinson, 1996) and stabilize the fly's gaze (Hengstenberg, 1991; Huston and Krapp, 2009). If the halteres are removed, flies are unable to maintain stable flight (Derham, 1714).

The halteres are imbued with hundreds of mechanosensory cells, the vast majority of which are campaniform sensilla (Chapman, 1982; Gnatzy et al., 1987). Although significant strides have been made in understanding how halteres influence flight behavior in flies (Bender and Dickinson, 2006; Dickinson, 1999; Mureli and Fox, 2015; Sherman and Dickinson, 2003), less is known about how the arrangement of the haltere campaniform sensilla influences their function, and which forces they may detect.

Furthermore, it is not known how the arrays of campaniform sensilla may differ among fly species. Diptera is the second-largest order of insects after Coleoptera (Grimaldi et al., 
2005), and contains species that exhibit a broad range of flight behaviors (Brodsky, 1994). Previous work has discussed haltere campaniform shape and arrangement for two highly-derived fly species: Calliphora (Calliphoridae: Gnatzy et al., 1987; Pflugstaedt, 1912), and Drosophila (Drosophilidae: Cole and Palka, 1982). These studies reported similar fields of campaniform sensilla and named them according to their relative locations (Fig 1A). Are these fields conserved across the entire order? How might they vary in shape, size, or placement? Because campaniform sensilla function by coupling deformation of a flexible cuticular dome to mechanical strain detected by an underlying mechanosensitive neuron, surface morphology and location will greatly impact the forces detectable by these structures. As a result, any variation we see in sensilla morphology or arrangement between fly species likely affects haltere function.

To better understand how the campaniform sensilla may detect inertial forces during flight in different species, we used scanning electron microscopy (SEM) to map and compare the arrays of campaniform sensilla of 150 flies from over 40 families across fly phylogeny, from the nematocera-type flies (the clades Tipulomorpha, Psychodomorpha, and Culicimorpha) to the calyptrates (Fig. 1B). We find distinct phylogenetic patterns of gross haltere morphology and sensilla arrangements, revealing a greater diversity of haltere morphology than previously documented and providing insight into the evolution and function of a complex, highly specialized organ.

\section{Methods}

\subsection{Sample collection}

Flies were collected from northeastern Ohio, New York City, and New Jersey. Other samples were taken from museum collections at the American Museum of Natural History and the Cleveland Museum of Natural History. All specimens were identified to family level, and a subset to genus and species levels (noted in table S1). Up to nine specimens were studied per family.

\subsection{SEM methods}

The halteres of all specimens were dissected from the body by cutting through the pleural sclerites in a ring around the base and then immersed in $95 \%$ ethanol overnight and allowed to air-dry. Halteres were mounted on SEM stubs (Ted Pella Inc., Redding, CA), coated with gold-palladium, and examined using either a Hitachi S4700 SEM (at the American Museum of Natural History) or a Helios Nanolab 650 SEM (at Case Western Reserve University).

\subsection{Gross measurements}

Gross measurements of halteres (e.g., haltere and bulb length) were made either from SEM images or with a Zeiss stereoscope outfitted with a digital stage micrometer. Bulb length of the haltere was defined as the length from the tip of the haltere to the point where the haltere expands from the stalk. In many of these specimens, it was not possible to accurately measure body length due to deformations upon drying.

\subsection{Quantifying row arrangements}

We digitized the position of the centroid of each campaniform sensillum using custom software written in Matlab (The Mathworks, Natick, MA). To estimate the linearity of the 
rows of sensilla that we observed, we sorted the sensilla into rows and measured the distance between adjacent sensilla. The total distance for the row of sensilla was divided by the straight-line distance between the first and last sensilla. Using this calculation, a row of campaniform sensilla that were aligned in a perfectly straight row would have a score of 1 , the minimum possible score, and a row with less alignment would have a higher score.

\subsection{Phylogenetic analysis}

We used a family-level chronogram from Wiegmann et al. (2011) to account for phylogenetic relationships. The tree was pruned to include only the taxa for which we had morphological data. We calculated Blomberg's $K$ (Blomberg et al., 2003) to test for phylogenetic signal, which is a measure of the extent to which trait variation between taxa may result from their phylogenetic relatedness, using the function "phylosig" from the R package phytools (Revell, 2012). Under Brownian motion (BM) trait evolution (traits evolve under a random walk at a constant rate), the expected value of $K$ is 1 (Ackerly, 2009), and significant phylogenetic signal is assessed by contrasting $K$ with that generated by randomizing trait values at the tips $(n=1000)$.

\section{Results}

\subsection{Gross haltere shape}

Representative images of some of the halteres we examined can be seen in Fig. 2. The longest halteres we examined belonged to Tipulidae, ranging from 1-3 $\mathrm{mm}$ in length (Fig. $2 \mathrm{~L}$, left). The shortest halteres were found in Scatopsidae $(0.17-0.18 \mathrm{~mm})$. Haltere length correlates at least in part with body size: two culicid species, Aedes aegypti and Toxorhynchites amboinensis, differ dramatically in size, and the larger Tx. amboinensis has longer halteres.

We also examined bulb length and the proportion of the bulb to the rest of the haltere (Fig. 2B, middle and right). Bulb length generally correlates with haltere length longer halteres have longer bulbs. However, we found that among the Neodiptera (with the exception of the Calyptratae), a reduction in the haltere stalk length resulted in the bulb occupying a greater proportion of the haltere, typically more than $40 \%$. Only in the basal families (Tipulidae, Trichoceridae, Ptychopteridae, and Blephariceridae) and the more distantly derived families of the Calyptratae (Muscidae, Calliphoridae, Sarcophagidae, Anthomyiidae, and Tachinidae) do we consistently observe flies with a long stalk and a small bulb (Fig. 2B, right). The similarity in gross haltere shape occurs despite the dramatically different body shapes of these two groups of flies: nematocerans are typically gracile with long, slender abdomens and legs, and the calyptrates are very stout-bodied.

Tests for phylogenetic signal found that all three quantities, haltere length, bulb length, and bulb length as a fraction of haltere length, varied significantly with phylogenetic relatedness (respectively: $K=1.13, p=0.002 ; K=1.04, p=0.003 ; K=$ $0.77, p=0.005$ ). An examination of the continuous trait maps (Fig. S1) reveals that the lower phylogenetic signal of the bulb length as a fraction of haltere length could be due to the convergent evolution of lengthened stalks in the nematocerans and calyptrates.

\subsection{Trends in campaniform numbers and density}


We next counted and characterized the sensilla present in each of the six fields (Fig. 3). Dipterans of the Eremoneura clade have much more stereotyped numbers of sensilla, with nearly all families possessing fewer sensilla than other dipterans. This trend is most noticeable among the dorsal flanking sensilla (dFS): the nematocerans, bibionomorphans, and orthoraphans all demonstrate variable numbers of dFS, but among the Eremoneura, all species studied possess at most only one dFS. Surprisingly, we found that most nematocerans, especially the Tipulomorpha and Psychodomorpha, entirely lacked the dorsal Hicks papillae (dHP). Among the nematocera, only blepharicerids appear to have any dHP (see section 3.3.4 below for a more detailed description). With the exception of the ventral Hicks papillae, the numbers of sensilla in all fields displayed a significant phylogenetic signal close to one, the expectation under a constant rate, random walk, BM process (dHP: $K=0.86, p=0.008$; dBP: $K=1.05, p=0.005$; dSP: $K=1.04, p=0.001$; vHP: $K=0.57, p=0.21$; vSP: $K=0.87, p=0.006$; dFS: $K=1.05, p=0.011$ ).

Additionally, we measured the density of campaniform sensilla within the three dorsal fields (Fig. 4A). Some families (Chironomidae and Ephydridae) had relatively high densities of dorsal basal plate (dBP) sensilla. Among dorsal scapal plate (dSP) sensilla, the density of campaniform sensilla is consistent and varies little across taxa. We did not find that the densities on the dorsal fields varied significantly with phylogeny (dHP: $K=0.48, p=0.42$; dBP: $K=0.59, p=0.21$; dSP: $K=0.43, p=0.58$ ).

Because there are no major variations in density in our data set, the primary determinant of sensilla number in any field is likely the size of the haltere. We find that sensilla number is indeed significantly correlated with haltere length in the three largest fields (dBP, dSP, and vSP; Fig. 4B). The correlation was similar for all three fields $\left(\mathrm{r}^{2}=\right.$ 0.39 to $0.41 ; p<0.001$ ). Tipulid flies fell considerably below the correlation line, with a low number of campaniform sensilla relative to their haltere length. Asilidae, Therevidae, and Tabanidae had high numbers of campaniform sensilla relative to length.

\subsection{Comparisons of sensilla morphology and major fields between families}

For all sensilla fields, we found more variability in the morphology of individual campaniform sensilla than had been previously documented. Previous studies focused on a few schizophoran flies (Cole and Palka, 1982; Gnatzy et al., 1987; Pringle, 1948; Smith, 1969), and much of the variation in individual campaniform shapes disappears after the emergence of the Asiloidea (a major sister group to the Eremoneura (Wiegmann et al., 2011)). Furthermore, the arrangement of sensilla in most fields appears to transition from variable in organization and orientation in nematocerans to uniform and organized in the Eremoneura. Below, we describe our findings for each field in greater detail.

\subsubsection{Scapal plate sensilla}

The dorsal and ventral scapal plate fields are the largest sensilla fields on the haltere. Overall, we found these two plates to be very similar in both the shape of their campaniform sensilla and their arrangements (dorsal scapal plates from several families are shown in Fig. 5; see Fig. S2 for images of ventral scapal plates). The overall shape of the scapal plates differs slightly: the dorsal scapal plate twists as it connects to the basal plate and the ventral scapal plate is generally flat.

As mentioned above, two trends are immediately noticeable: foremost, sensilla shape is much more variable than previously described. Previous studies focusing on 
schizophoran flies noted neat arrangements of sensilla in rows in which adjacent sensilla are fused, such that the cuticular cap of the campaniform sensilla is flanked by two square caps (Fig. 5G-J). However, we found that the scapal plate sensilla of many dipteran species outside the Eremoneura typically consists of two cuticular caps flanking a central dome, and the caps of adjacent sensilla are not fused. These cuticular caps vary in their shape: some are extremely conical, as in Culicidae (Fig. 5E), whereas others are more irregular in shape, as in Psychodidae (Fig. 5D). The space between these cuticular caps also varies - the flanking caps of culicids (Fig. 5E) are extremely close to one another, whereas those of conopids (Fig. 5I) are fairly far apart. Surprisingly, some specimens even have multiple shapes present in the same field, such as Trichoceridae (Fig. 5A) and Tipulidae (Fig. 5B). Anterior to the "typical" dSP sensilla, but posterior and proximal to the flanking sensilla, are sensilla with a high socket and cap (Fig. 5, white arrows). We consider these sensilla to comprise an "extra field" since they are not clearly part of any previously described field. The proximal dSP sensilla are also more recessed than the distal dSP sensilla, but still have a shape similar to the distal dSP sensilla (Fig. 5, yellow arrows).

The second trend we noticed was that sensilla arrangement varied in its linearity across dipterans. Among nematocerans, the dSP sensilla are often oriented in multiple directions and clear rows are difficult to discern (Fig. 5C). However, in Brachycera, and especially Cyclorrhapha, dSP sensilla fuse into rows that are oriented roughly perpendicular to the long axis of the haltere (Figs. 5E-J). Based on measurements of linearity of sensilla arrangement (Fig. 6), we obtained a strong phylogenetic signal $(K=$ $1.33, p=0.004)$.

\subsubsection{Flanking sensilla}

Flanking sensilla are typically located in the furrow immediately anterior to the dorsal scapal plate (Fig. 7). The shape of these sensilla is generally conserved across all dipterans, and consists of a high-profile, circular campaniform surrounded by a socket (Fig. 7A-G). Some appear to be more ovular than others, and some have a collar with or without a break, but compared to sensilla in other fields, shape variation is slight. However, number and placement of these sensilla does vary from family to family. Basal dipterans have anywhere from zero to nine flanking sensilla, but by the emergence of Dolichopodidae, all Eremoneura families examined have a single or absent flanking sensillum. The location of the sensilla varies across all Diptera (Fig. 7H-N). For example, all species of Culicidae have flanking sensilla that are located much further from the dorsal scapal plate and closer to the ventral scapal plate and no longer in a furrow (Fig. 7I, arrow). In Conopidae, the flanking sensillum is located distal to the dorsal scapal plate sensilla (Fig. 7L). If a fly possesses multiple flanking sensilla, they are almost always arranged in a straight row (Fig. 7D-G), except in Athericidae (Fig. 7A).

\subsubsection{Basal plate sensilla}

The basal plate sensilla have the general morphology of the canonical, dome-shaped campaniform sensilla found in all insects (Fig. 8) (Keil, 1997). Variations in shape were small, but notable: some sensilla had a higher profile (Fig. 8B, C), and some were much more ovular (Fig. 8G-J). Ovular basal plate sensilla, which are thought to be directionally sensitive (Fox and Daniel, 2008; Smith, 1969; Thurm et al., 1974; Zill and Moran, 1981), 
were only seen in the Brachycera; the sensilla of nematocerans were much more circular and uniform (Fig. 8A-E).

The most striking observed difference between the basal plates of various fly species is the arrangement of the sensilla. The basal plate sensilla of Brachycera are organized into obvious rows, with microtrichia between the rows draping over the campaniform domes (Fig. 8F-J). Many dipterans outside the Eremoneura clade lack these highly linear rows, and the microtrichia are instead arranged hexagonally around the campaniform domes (Fig. 8A) or are completely absent (Fig. 8C). As with dSP, we analyzed the linearity of dBP sensilla arrangement, and found an even higher phylogenetic signal than that calculated for dSP linearity $(\mathrm{K}=1.87, p=0.001)$ (Fig. 9).

We also observed that the basal plate of some families, like Culicidae (Fig. 8C), was much more contoured than the flat basal plate of the majority of flies. Although it is possible that this is an artifact of the drying process, we noted it in all culicid specimen despite being preserved in a variety of ways (freshly-caught, stored dry, stored in the freezer, or stored in alcohol).

\subsubsection{Hicks papillae}

Representative images of the dorsal Hicks papillae (dHP) can be seen in Fig.10. Ventral Hicks papillae can be seen in Fig. S3. Most nematocerans lack dorsal Hicks papillae but still have ventral Hicks papillae. Blepharicerids are the only nematocerans that possess dHP (Fig. 10A). However, in the blepharicerids, the number, arrangement, and location of these sensilla, sandwiched between two groups of lens-shaped, dorsal basal plate (dBP)-like sensilla (see arrows, Fig. 10A), suggest that this field evolved independently from the dHP of other dipterans. After the emergence of dHP among Brachycera, the number of sensilla in the field decreases to only a few, such as in Ephydridae (Fig. 3). In calyptrate flies, sensilla number again increases, and they exhibit a distinctive field structure consisting of two straight rows (Figs. 10K, S4).

The Hicks papillae show substantial variation in morphology, from the canonical shape previously described (i.e. Fig. 10I), to swollen bacilliform (Fig. 10G), long vermiform (Fig. 10B), shallow depressions (Fig. 10E), or reniform structures (Fig. 10F). Hicks papillae are also recessed to varying degrees, in which the dome and surrounding projections of the cuticle are inserted into the haltere's surface.

\section{Discussion}

Neural information from haltere campaniform sensilla is essential for fly flight, and these tiny organs have fascinated scientists for centuries (Derham, 1714). Nevertheless, little is known about the mechanisms by which campaniform sensilla and their associated neurons detect forces acting on the haltere and send this crucial information to the central nervous system. Previous studies indicated that the physical shape and location of the campaniform sensilla, rather than neural specialization or processing, are the main determinants of the information in the haltere nerve (Fox and Daniel, 2008; Fox et al., 2010). Given the importance of sensory cell structure to force sensing, we have documented and quantified the arrangement of haltere sensilla over the dipteran phylogeny, uncovering a diversity of campaniform sensilla shape, number, and distribution with the aim of understanding the evolution of the haltere and ultimately, describing a mechanism for its function. 


\subsection{How do campaniform sensilla help the fly sense bodily forces?}

Campaniform sensilla occur on many locations on the bodies of all insects, particularly at the joints. As forces impinge on the cuticle, deformation of the sensilla dome stimulates the dendrites of nerve cells underneath the dome, sending action potentials down the axons and synapsing onto motor neurons and other neurons in the thoracic ganglia (Chan and Dickinson, 1996; Keil, 1997; Merritt and Murphey, 1992; Sane and McHenry, 2009; Smith, 1969). The neurons of the haltere campaniform sensilla are able to detect the forces resulting from small movements of the haltere, and they do this with high speed and high reliability over a broad range of haltere oscillation frequencies (Fox and Daniel, 2008). These broad responses to haltere deflections demonstrate that the haltere primary afferent neurons are not specialized to detect particular types or frequencies of forces. Therefore, the neural activity will be determined mostly by the mechanical forces that occur on the campaniform sensilla, and not by any neural filtering or processing. The arrangement and morphology of the campaniform sensilla, then, are the primary determinants of the sensory information that is sent via the haltere nerve to the central nervous system for controlling wing-steering (Dickinson, 1999; Fayyazuddin and Dickinson, 1996; Heide, 1983) and head movement (Hengstenberg, 1988; Huston and Krapp, 2009) behaviors. Therefore, we would expect that the sensory information available in the haltere nerves of Tipulidae might be very different from that in Calliphoridae or Drosophilidae, given their very different arrangements and morphologies of campaniform sensilla, and their different manners of flight.

However, it is not known precisely how campaniform shape and arrangement influence neural activity. Due to their small size and often inaccessible location, it is difficult to directly measure the strains experienced by campaniform sensilla (Blickhan and Barth, 1985; Sane and McHenry, 2009) and thus we have little empirical evidence of how campaniform shape or arrangement might filter or transform these strains. Numerical and analytical models have attempted to fill this gap. One model (Skordos et al., 2002) has demonstrated the importance of the shape and discontinuities of the cuticle surrounding the campaniform dome as the primary amplifier and filter of strain conveyed to the central mechanosensory neuron. Our data raise further questions about strain filtering through the campaniform dome. Is the lack of diversity of campaniform shapes in Eremoneura due to the evolution of campaniform shapes that are sensitive to specific forces? How important is directional selectivity for strain sensing? Furthermore, we observed a trend towards linear arrangement of campaniform sensilla in the more derived dipterans - how does linearity and field orientation affect the concentration and filtering of inertial forces on the haltere? More comprehensive models, combined with the data we show here, may reveal why certain fields consist of campaniform sensilla of any given type.

Current models of haltere neural encoding suggest that particular campaniform sensilla encode a specific phase of the haltere's sinusoidal oscillations with preciselytimed spikes (Fox et al., 2010). Based on this model, increased numbers of campaniform sensilla might result in increased temporal resolution and thus finer control over wingsteering maneuvers (Fayyazuddin and Dickinson, 1999). However, we did not observe a general trend towards an increased number of campaniform sensilla in more recently evolved clades. This trend is particularly apparent in the flanking sensilla, which vary in 
number from zero to 10 in nematocerans, but appear as a single sensillum in the majority of the Eremoneura families. We did notice that flies with unusually high sensilla numbers for their length, like Asilidae, include aerial predators, and those with unusually low numbers are nectar-feeding or non-feeding crane flies, suggesting that sensilla number may correlate to some degree with ecological demands and flight ability. Unfortunately, "flight ability" currently remains a poorly defined term, and there is little data comparing the flight behaviors of different dipteran species. Future work is necessary to quantify aspects of flight behavior and correlate these with sensilla arrangement.

\subsection{Do specific fields of campaniform sensilla provide specific types of mechanosensory information?}

Although the neural encoding properties of haltere primary afferent neurons have been measured (Fox and Daniel, 2008), the specific encoding properties of neurons emanating from particular fields have not, due to the difficulty of anatomically labeling individual neurons. Therefore, we must rely on our descriptions of campaniform sensilla morphology and neural anatomy (Chan and Dickinson, 1996) to predict how the fields might function.

The scapal plates on both the dorsal and ventral aspects of the haltere are oriented parallel to the haltere's long axis, and thus input from these fields may be able to report the haltere's oscillation in its natural stroke plane during straight flight (Fox and Daniel, 2008; Nalbach, 1993; Pringle, 1948). The anatomy of the scapal plates shown here raises more questions regarding this functionality: how does the coupling of adjacent sensilla into rows change sensitivity to small deviations in haltere oscillation? Do the variously oriented scapal plate sensilla of nematoceran dipterans, like in the tipulids, function differently from those of the cyclorraphan flies?

There is some evidence that the dorsal basal plate (dBP) is not activated by the oscillations that occur in straight flight, but may be activated when the fly experiences Coriolis forces associated with body rotations (Nalbach and Hengstenberg, 1986; Pringle, 1948; Thompson et al., 2009). Neurons from campaniform sensilla in dBP project to a wing-steering motor neuron (Chan and Dickinson, 1996), and synapse electrotonically onto it, enabling reflexive, rapid flight control (Fayyazuddin and Dickinson, 1996). The long axes of the campaniform sensilla in $\mathrm{dBP}$ are oriented approximately $30^{\circ}$ from the rows in which they are oriented in Calliphora (Chapman, 1982; Pringle, 1948). This orientation may make them more selective for off-axis forces that might occur during body rotations. We observed significant variation in the eccentricity of campaniform sensilla in this field (Fig. 8): nematocerans typically had rounder dBP sensilla compared to the highly ovate sensilla of many of the calyptrate flies. Are nematocerans as adept at sensing Coriolis forces using this field, despite their round sensilla? Or does the directional selectivity of ovate sensilla confer added sensitivity?

Notably, we found that most nematoceran dipterans lacked an entire field, the dorsal Hicks papillae. Its absence in these flies indicates that it is not required for haltere function, perhaps because it is partially redundant with the dorsal scapal plate (Chan and Dickinson, 1996). However, its existence in the majority of dipterans (some nematocerans and all Brachycera), and its unique shape in calyptrate flies, suggests that it may have a specific sensory function. 


\subsection{Distinguishing characteristics of the halteres of Calyptratae}

Many species of Calyptratae (Fig. 1) can be described as highly successful. These flies include cosmopolitan generalists like house flies, flesh flies, and blow flies. They are speciose (Wiegmann et al., 2011), common in many habitats, and are skilled fliers, able to move at high speeds and chase conspecifics on the wing (Land and Collett, 1974). Much of our understanding of haltere sensory physiology and function is based on the function of blowfly halteres (Fayyazuddin and Dickinson, 1996; Hengstenberg, 1991; Huston and Krapp, 2009), but we note several characteristics of calyptrate halteres that distinguish them from the halteres of other fly species. First, they have long stalks and small bulbs (Fig. 2), which is not observed in other cyclorrhaphan flies. Second, they have two straight rows of dorsal Hicks papillae (Fig. 9K), a unique arrangement. Finally, other studies have observed that calyptrate flies rapidly oscillate their halteres while walking as well as in flight (Fraenkel, 1939; Hall et al., 2015; Miller, 1977; Sandeman and Markl, 1980). Sarcophaga has been shown to use its halteres during vertical walking behavior to stabilize itself against perturbations (Hall et al., 2015). These findings suggest that the Calyptratae may use their halteres in uniquely adaptive ways to guide multiple behaviors, and that perhaps some of their success as a clade can be attributed to their haltere function.

\subsection{Other haltere mechanosensory structures}

The major sensory cells on the halteres are the campaniform sensilla, and the axons of the neurons emanating from the campaniform sensilla comprise the bulk of the haltere nerve. However, there are a small number of other sensory cells on the haltere. Our analysis does not include any comparisons of the chordotonal organs or the bristles at the base and knob of the haltere (Pflugstaedt, 1912). The chordotonal organs lie inside the haltere (Chapman, 1982) and have no surface morphology. As a result, any examination of the chordotonal organ and how it may vary between species would require a different (i.e. histological) approach from the scanning electron microscopy used here. We also do not describe the presence and arrangement of mechanosensory bristles. We consider the bristles to be outside the scope of our study: the haltere is a proprioceptive structure, and thus characterization of proprioceptive mechanoreceptors like the campaniform sensilla, versus exteroreceptors like the bristles (Newland and Burrows, 1997), will more likely inform an understanding of the haltere's function.

\subsection{The haltere is a highly specialized and derived proprioceptive organ}

There is no generally recognized intermediate organ between the ancestral hindwing and the derived haltere, and no extant insects have haltere-like hindwings besides the true flies, although various unrelated insects have highly reduced hind wings (e.g., male Coccoidea and the Mesozoic scorpionflies Parapolycentropus). An analogous organ is the forewings of the Strepsiptera, which serve a similar function as mechanosensory gyroscopes but are not homologous to the halteres (Pix et al., 1993). These organs are shaped similarly to the club-like halteres but only have a single field of 70-100 campaniform sensilla at the base on both the dorsal and ventral sides. Unlike the flies, the Strepsiptera have a plate of bristles, known as a hair plate, near the base of the halterelike structure, and thus they may use the campaniform sensilla to detect lateral forces while monitoring the forewing oscillation phase with the hair plate (Pix et al., 1993). 
Subsequent research has found that other insect appendages may be able to act as gyroscopes to sense body rotations. Any appendage experiencing oscillation in a single plane while rotating in a second plane would experience Coriolis forces, and the presence of campaniform, scolopidial, or other mechanosensory organs at body joints makes nearly all insect appendages candidates for gyroscopic sensing. The antennae (Sane et al., 2007) and wings (Dickerson et al., 2014; Eberle et al., 2015) of moths have been shown to mediate some responses to body rotations during flight.

The lack of intermediate halteres, and a general conservation of field organization across Diptera, suggests that a basic sensilla landscape became fixed quite early. The major fields of campaniform sensilla on the wings are serial homologues of the fields of sensilla on the wing (Chan and Dickinson, 1996; Cole and Palka, 1982; Gnatzy et al., 1987; Palka et al., 1979), and the flanking sensilla may be the remnants of the ten distal sensilla on the wing blade (Chan and Dickinson, 1996).

We found that dipterans outside the Eremoneura demonstrate a high degree of variability in the shape, number, and arrangement of their campaniform sensilla. Among the Eremoneura, the variability in sensilla is decreased, and in the Calyptratae, all flies possess an intermediate number of campaniform sensilla arranged into straight rows. Overall, our analysis reveals a previously undocumented variety of haltere shape, campaniform shape, and campaniform arrangement that arose over the course of fly evolution, and which will likely inform any future studies of haltere function.

\section{Acknowledgements}

We thank Nanthanwan Avishai and the Swagelok Center for Surface Analysis of Materials for assistance with scanning electron microscopy at Case Western Reserve University. We also thank Gabriella Wolff, Chloé A. Lahondère, and the Cleveland Museum of Natural History for access to specimens, Tom Daniel for his support and comments, and Jessica Arbour for her assistance with phylogenetic analyses. This work was supported by laboratory start-up funds from Case Western Reserve University and Air Force Office of Scientific Research grants [FA9550-14-0398 and FA9550-16-10165] to JLF, an NSF-funded REU at AMNH (PI Mark Siddall), and an NSF grant to DG [OPUS DEB 1556502].

Ackerly, D., 2009. Conservatism and diversification of plant functional traits:

Evolutionary rates versus phylogenetic signal. Proc. Natl. Acad. Sci. U. S. A. 106 Suppl , 19699-706. doi:10.1073/pnas.0901635106

Bender, J.A., Dickinson, M.H., 2006. A comparison of visual and haltere-mediated feedback in the control of body saccades in Drosophila melanogaster. J Exp Biol 209, 4597-4606. doi:10.1242/jeb.02583

Blickhan, R., Barth, F.G., 1985. Strains in the exoskeleton of spiders. J Comp Physiol A 157, 115-147. doi:10.1007/BF00611101

Blomberg, S. P., T. Garland, and A. R. Ives. 2003. Testing for phylogenetic signal in comparative data: behavioral traits are more labile. Evolution 57:717-745.

Brodsky, A.K., 1994. The evolution of insect flight. Oxford University Press, Oxford.

Chan, W.P., Dickinson, M.H., 1996. Position-specific central projections of mechanosensory neurons on the haltere of the blow fly, Calliphora vicina. J Comp 
Neurol 369, 405-418. doi:10.1002/(SICI)1096-9861(19960603)369:3\&1t;405::AIDCNE6\&gt;3.0.CO;2-9

Chapman, R.F., 1982. The insects : structure and function.

Cole, E.S., Palka, J., 1982. The pattern of campaniform sensilla on the wing and haltere of Drosophila melanogaster and several of its homeotic mutants. J Embryol Exp Morphol 71, 41-61.

Derham, W., 1714. Physico-theology (Boyle lecture for 1711). W. Innys, London.

Dickerson, B.H., Aldworth, Z.N., Daniel, T.L., 2014. Control of moth flight posture is mediated by wing mechanosensory feedback. J Exp Biol 217, 2301-2308. doi:10.1242/jeb.103770

Dickinson, M.H., 1999. Haltere-mediated equilibrium reflexes of the fruit fly, Drosophila melanogaster. Philos Trans R Soc L B Biol Sci 354, 903-916. doi:10.1098/rstb.1999.0442

Eberle, A.L., Dickerson, B.H., Reinhall, P.G., Daniel, T.L., 2015. A new twist on gyroscopic sensing: body rotations lead to torsion in flapping, flexing insect wings. J R Soc Interface 12, 20141088. doi:10.1098/rsif.2014.1088

Fayyazuddin, A., Dickinson, M.H., 1999. Convergent mechanosensory input structures the firing phase of a steering motor neuron in the blowfly, Calliphora. J Neurophysiol 82, 1916-1926.

Fayyazuddin, A., Dickinson, M.H., 1996. Haltere afferents provide direct, electrotonic input to a steering motor neuron in the blowfly, Calliphora. J Neurosci 16, 52255232.

Fox, J.L., Daniel, T.L., 2008. A neural basis for gyroscopic force measurement in the halteres of Holorusia. J Comp Physiol A 194, 887-897. doi:10.1007/s00359-0080361-z

Fox, J.L., Fairhall, A.L., Daniel, T.L., 2010. Encoding properties of haltere neurons enable motion feature detection in a biological gyroscope. Proc Natl Acad Sci USA 107, 3840-3845. doi:10.1073/pnas.0912548107

Fraenkel, G., 1939. The Function of the Halteres of Flies (Diptera). Proc Zool Soc London A109, 69-78. doi:10.1111/j.1096-3642.1939.tb00049.x

Gnatzy, W., Grünert, U., Bender, M., 1987. Campaniform sensilla of Calliphora vicina (Insecta, Diptera). I. Topography. Zoomorphology 106, 312-319.

Grimaldi, David A., and Michael S. Engel. 2005. Evolution of the insects. Cambridge [U.K.]: Cambridge University Press.

Hall, J.M., McLoughlin, D.P., Kathman, N.D., Yarger, A.M., Mureli, S., Fox, J.L., 2015. Kinematic diversity suggests expanded roles for fly halteres. Biol Lett 11, 20150845. doi:10.1098/rsbl.2015.0845

Heide, G., 1983. Neural mechanisms of flight control in Diptera, in: Nachtigall, W. (Ed.), BIONA-Report 2. G. Fischer, Stuttgart, pp. 35-52.

Hengstenberg, R., 1991. Gaze control in the blowfly Calliphora: a multisensory, twostage integration process. Semin Neurosci 3, 19-29. doi:http://dx.doi.org/10.1016/1044-5765(91)90063-T

Hengstenberg, R., 1988. Mechanosensory control of compensatory head roll during flight in the blowfly Calliphora erythrocephala Meig. J Comp Physiol A 163, 151-165. doi:10.1007/BF00612425

Huston, S.J., Krapp, H.G., 2009. Nonlinear integration of visual and haltere inputs in fly 
neck motor neurons. J Neurosci 29, 13097-13105. doi:10.1523/JNEUROSCI.291509.2009

Keil, T., 1997. Functional morphology of insect mechanoreceptors. Microsc Res Tech 39, 506-531.

Land, M.F., Collett, T.S., 1974. Chasing behaviour of houseflies (Fannia canicularis). J Comp Physiol 89, 331-357. doi:10.1007/BF00695351

Merritt, D.J., Murphey, R.K., 1992. Projections of leg proprioceptors within the CNS of the fly Phormia in relation to the generalized insect ganglion. J Comp Neurol 322, 16-34. doi:10.1002/cne.903220103

Miller, P.L., 1977. Haltere activity in a flightless hippoboscid fly, Crataerina pallida. J Insect Physiol 23, 855-860. doi:http://dx.doi.org/10.1016/0022-1910(77)90010-5

Mureli, S., Fox, J.L., 2015. Haltere mechanosensory influence on tethered flight behavior in Drosophila. J Exp Biol 218, 2528-37. doi:10.1242/jeb.121863

Nalbach, G., 1993. The halteres of the blowfly Calliphora. J Comp Physiol A 173, 293300. doi:10.1007/BF00212693

Nalbach, G., Hengstenberg, R., 1986. Die Halteren von Calliphora als Drehsinnesorgan. Verh Dtsch Zool Ges.

Newland, P.L., Burrows, M., 1997. Processing of tactile information in neuronal networks controlling leg movements of the locust. J. Insect Physiol. 43, 107-123. doi:10.1016/S0022-1910(96)00081-9

Palka, J., Lawrence, P.A., Hart, H.S., 1979. Neural projection patterns from homeotic tissue of Drosophila studied in bithorax mutants and mosaics. Dev Biol 69, 549575. doi:10.1016/0012-1606(79)90311-7

Pflugstaedt, H., 1912. Die halteren der dipteren. Z Wiss Zool 100, 1-59.

Pix, W., Nalbach, G., Zeil, J., 1993. Strepsipteran forewings are haltere-like organs of equilibrium. Naturwissenschaften 80, 371-374. doi:10.1007/BF01138795

Pringle, J.W.S., 1948. The gyroscopic mechanism of the halteres of Diptera. Philos Trans R Soc L B Biol Sci 233, 347-384. doi:10.1098/rstb.1948.0007

Revell, L.J., 2012. phytools: An R package for phylogenetic comparative biology (and other things). Methods Ecol. Evol. 3, 217-223. doi:10.1111/j.2041210X.2011.00169.x

Sandeman, D.C., Markl, H., 1980. Head movements in flies (Calliphora) produced by deflexion of the halteres. J Exp Biol 85, 43-60.

Sane, S.P., Dieudonné, A., Willis, M.A., Daniel, T.L., 2007. Antennal Mechanosensors Mediate Flight Control in Moths. Science 315, 863-866. doi:10.1126/science. 1133598

Sane, S.P., McHenry, M.J., 2009. The biomechanics of sensory organs. Integr Comp Biol 49, i8-i23. doi:10.1093/icb/icp112

Sherman, A., Dickinson, M.H., 2003. A comparison of visual and haltere-mediated equilibrium reflexes in the fruit fly Drosophila melanogaster. J Exp Biol 206, 295302. doi: $10.1242 /$ jeb. 00075

Skordos, A., Chan, P.H., Vincent, J.F. V, Jeronimidis, G., 2002. A novel strain sensor based on the campaniform sensillum of insects. Philos Trans A Math Phys Eng Sci 360, 239-53. doi:10.1098/rsta.2001.0929

Smith, D.S., 1969. The fine structure of haltere sensilla in the blowfly Calliphora erythrocephala (Meig.), with scanning electron microscopic observations on the 
haltere surface. Tissue Cell 1, 443-484.

Thompson, R.A., Wehling, M.F., Evers, J.H., Dixon, W.E., 2009. Body rate decoupling using haltere mid-stroke measurements for inertial flight stabilization in Diptera. J Comp Physiol A 195, 99-112. doi:10.1007/s00359-008-0388-1

Thurm, U., Stedtler, A., Foelix, R., 1974. Reizwirksame Verformungen der Terminalstrukturen eines Mechanorezeptors. Verh Dtsch Zool Ges 67, 4700-4710. Weisstein, E.W., n.d. Eccentricity.

Wiegmann, B.M., Trautwein, M.D., Winkler, I.S., Barr, N.B., Kim, J.-W., Lambkin, C., Bertone, M.A., Cassel, B.K., Bayless, K.M., Heimberg, A.M., Wheeler, B.M., Peterson, K.J., Pape, T., Sinclair, B.J., Skevington, J.H., Blagoderov, V., Caravas, J., Kutty, S.N., Schmidt-Ott, U., Kampmeier, G.E., Thompson, F.C., Grimaldi, D.A., Beckenbach, A.T., Courtney, G.W., Friedrich, M., Meier, R., Yeates, D.K., 2011. Episodic radiations in the fly tree of life. Proc Natl Acad Sci USA 108, 5690-5695. doi:10.1073/pnas.1012675108

Zill, S.N., Moran, D.T., 1981. The exoskeleton and insect proprioception. I. Responses of tibial campaniform sensilla to external and muscle-generated forces in the American cockroach, Periplaneta americana. J Exp Biol 91, 1-24. 


\section{Figure Legends:}

Figure 1. Haltere morphology and sensilla were examined in $\mathbf{4 2}$ families spanning the dipteran order. (A) Schematic of the haltere and its major sensilla fields. Halteres are the modified hind wings of dipterans. Sensilla are clustered into four fields on the dorsal side: dorsal Hicks papillae (dHP), dorsal basal plate sensilla (dBP), dorsal scapal plate sensilla (dSP), and the flanking sensilla (FS). On the ventral side, they are clustered in either the ventral Hicks papillae (vHP) or the ventral scapal plate sensilla (vSP). (B) Phylogenetic tree (adapted from Wiegmann, 2011) showing the families we studied along with their clade. 1-9 specimens were studied per family, not necessarily all from the same species.

Figure 2. Dipterans show large variation in gross haltere morphology. (A-K) Representative SEM images of haltere shape from a subset of families. Scale bar denotes $100 \mu \mathrm{m}$. (L) From left to right: haltere length, bulb length, and the ratio of bulb length to haltere length across the dipteran order. Each data point represents a measurement taken from a haltere. Dotted line denotes a bulb length/haltere length ratio of 0.4. Tx.: Toxorhynchites amboinensis; Aed.: Aedes aegypti

Figure 3. Nematocera and orthrorhaphan Brachycera show greater diversity in sensilla numbers in each field. Scatterplots show the number of sensilla counted per field across the different dipteran families. Each data point represents a value taken from one haltere. Fields counted include (top to bottom): dorsal Hicks papillae (dHP), dorsal basal plate (dBP), dorsal scapal plate (dSP), ventral Hicks papillae (vHP), ventral scapal plate (vSP) and flanking sensilla (flanking).

Figure 4. Sensilla density, though variable, does not strongly correlate with phylogenetic position. (A) Scatterplot shows the sensilla density calculated across dipteran families for the three dorsal fields: dorsal Hicks (dHP), dorsal basal plate (dBP), and dorsal scapal plate (dSP). Multiple specimens are shown for several families. (B) Scatterplot of sensilla number per indicated field as a function of haltere length. Linear regression analysis indicates that campaniform sensilla number is significantly correlated with haltere length for each field measured $(\mathrm{p}<0.001)$. Tx.: Toxorhynchites amboinensis; Aed.: Aedes aegypti

Figure 5. Dorsal scapal plate sensilla. Representative SEM images of dorsal scapal plate sensilla from selected families. Scale bar denotes $10 \mu \mathrm{m}$. (A, B) white arrows point to the extra sensilla field not previously described. (A-C) yellow arrows point to sensilla at the proximal end of the field that are shallower and differently oriented from the sensilla at the distal end of the field.

Figure 6. Linearity scores for the dorsal scapal field correlate with relatedness. Linearity is measured by dividing the total distance between campaniform sensilla in a given row by the straight-line distance between the first and last sensilla. Campaniform sensilla arranged in a perfectly straight line would therefore have a low linearity score (minimum 1), and higher scores indicate less straight rows. Linearity was measured for 
multiple rows of campaniform sensilla in each specimen, and means for each family were used to construct trait map shown above. Continuous trait maps were generated using the function "contmap" in the R package phytools by incorporating a phylogeny (Wiegmann et al., 2011) and interpolating branch lengths based on the age of nodes and their estimated ancestral value under Brownian motion evolution (see Fig. 6). Insets: SEMs of dSP from Tipulidae (blue) and Anthomyidae (red) showing positions of campaniform sensilla as digitized for this analysis (white dots/lines).

Figure 7. Flanking sensilla. Representative SEM images of dorsal flanking sensilla from a subset of families. (A-G) Detail of some of the variation seen in flanking sensilla shape. Scale bar denotes $5 \mu \mathrm{m}$. (H-N) Images demonstrating variety in flanking sensilla positioning. Arrow points to flanking sensilla. Scale bar denotes $10 \mu \mathrm{m}$. Blepha: Blephariceridae.

Figure 8. Basal plate sensilla. Representative SEM images of basal plate sensilla from a subset of families. Scale bar denotes $10 \mu \mathrm{m}$.

Figure 9. Linearity scores for dorsal basal plate sensilla correlate with relatedness. Linearity is measured by dividing the total distance between campaniform sensilla in a given row by the straight-line distance between the first and last sensilla. Campaniform sensilla arranged in a perfectly straight line would therefore have a low linearity score (minimum 1), and higher scores indicate less straight rows. Linearity was measured for multiple rows of campaniform sensilla in each specimen, and means for each family were used to construct trait map shown above. Continuous trait maps were generated using the function "contmap" in the R package phytools by incorporating a phylogeny (Wiegmann et al., 2011) and interpolating branch lengths based on the age of nodes and their estimated ancestral value under Brownian motion evolution (see Fig. 6). Insets: SEMs of dSP from Tipulidae (blue) and Anthomyidae (red) showing positions of campaniform sensilla as digitized for this analysis (white dots/lines).

Figure 10. Dorsal Hicks papillae. Representative SEM images of dorsal Hicks papillae from a subset of families. Scale bar denotes $10 \mu \mathrm{m}$. (A) arrows point to lens-shaped sensilla on either side of the dHP. 
Dorsal

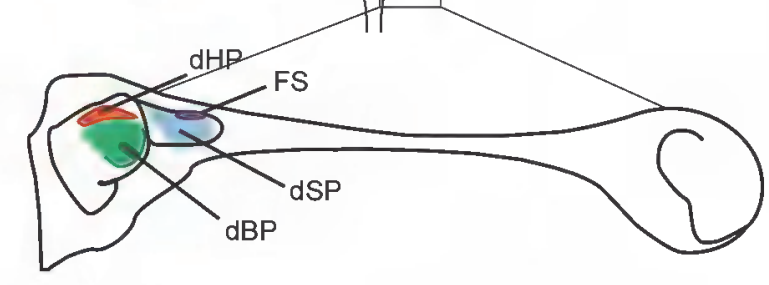

Ventral

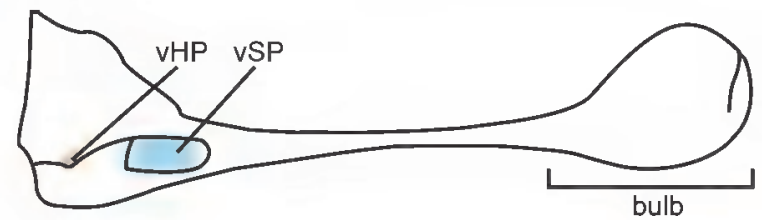

B

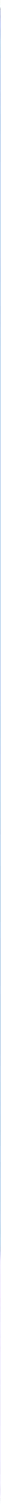

Representative family

Anthomyiidae

Calliphoridae

uscidae

Ephydridae

Chamaemyiidae

Heleomyzidae

Conopidae

Micropezidae

Chloropidae

Lonchopteridae

Phoridae

Dolichopodidae

Asilidae

Bombyliidae

Tabanidae

Rhagionidae

xylophagidae

Sciaridae

Bibionidae

Scatopsidae

Anisopodidae

Chironomidae

Culicidae

Psychodidae Blephariceridae

tychopteridae

Trichoceridae
Tachinidae

Sarcophagidae

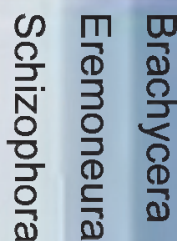



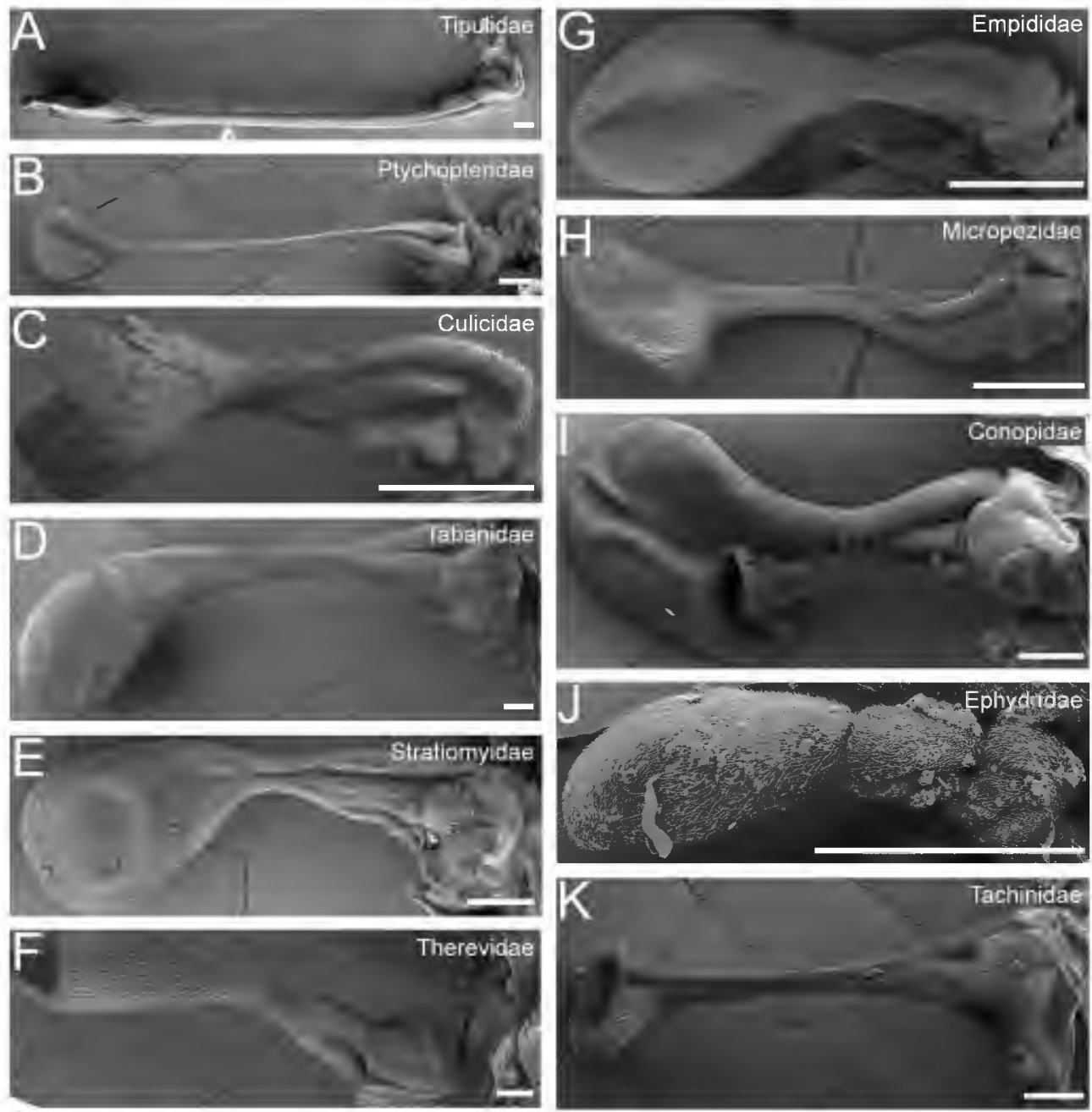

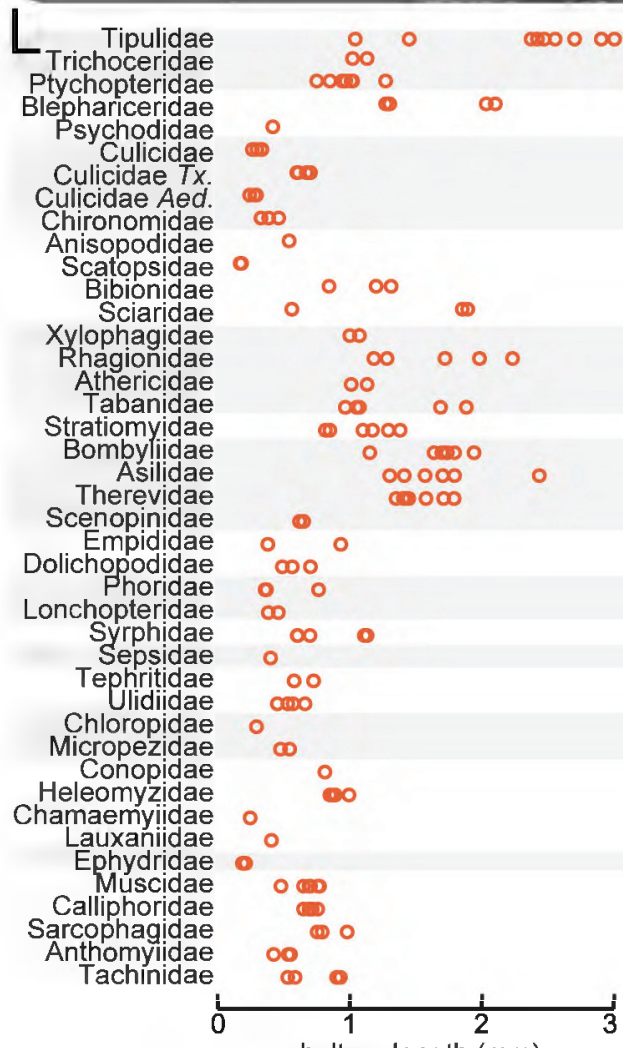
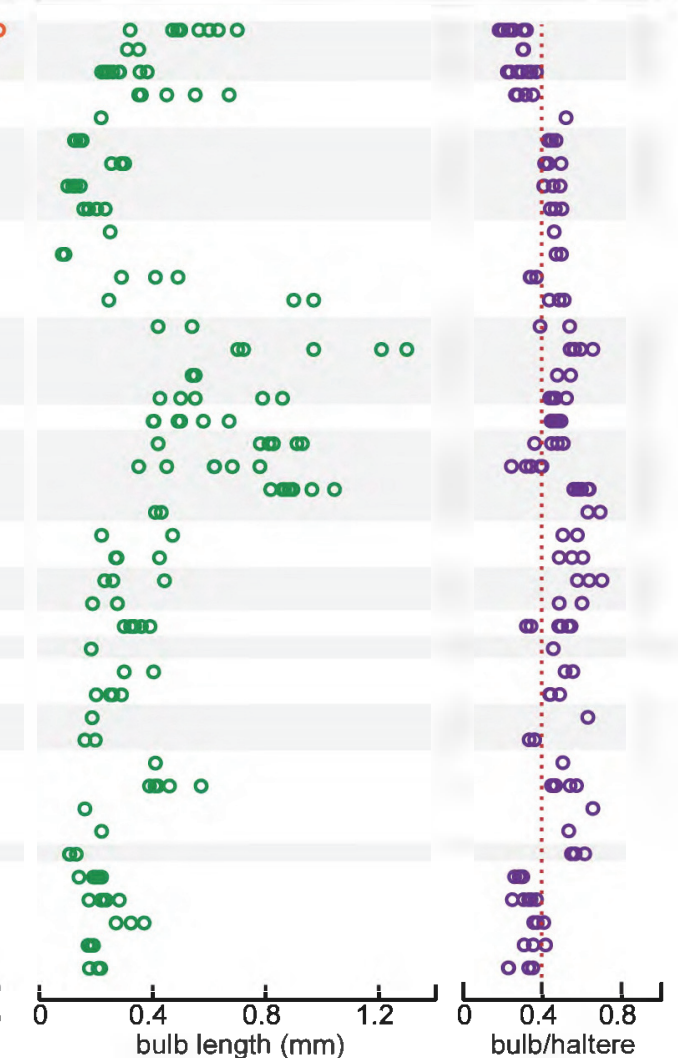
campaniform number by field

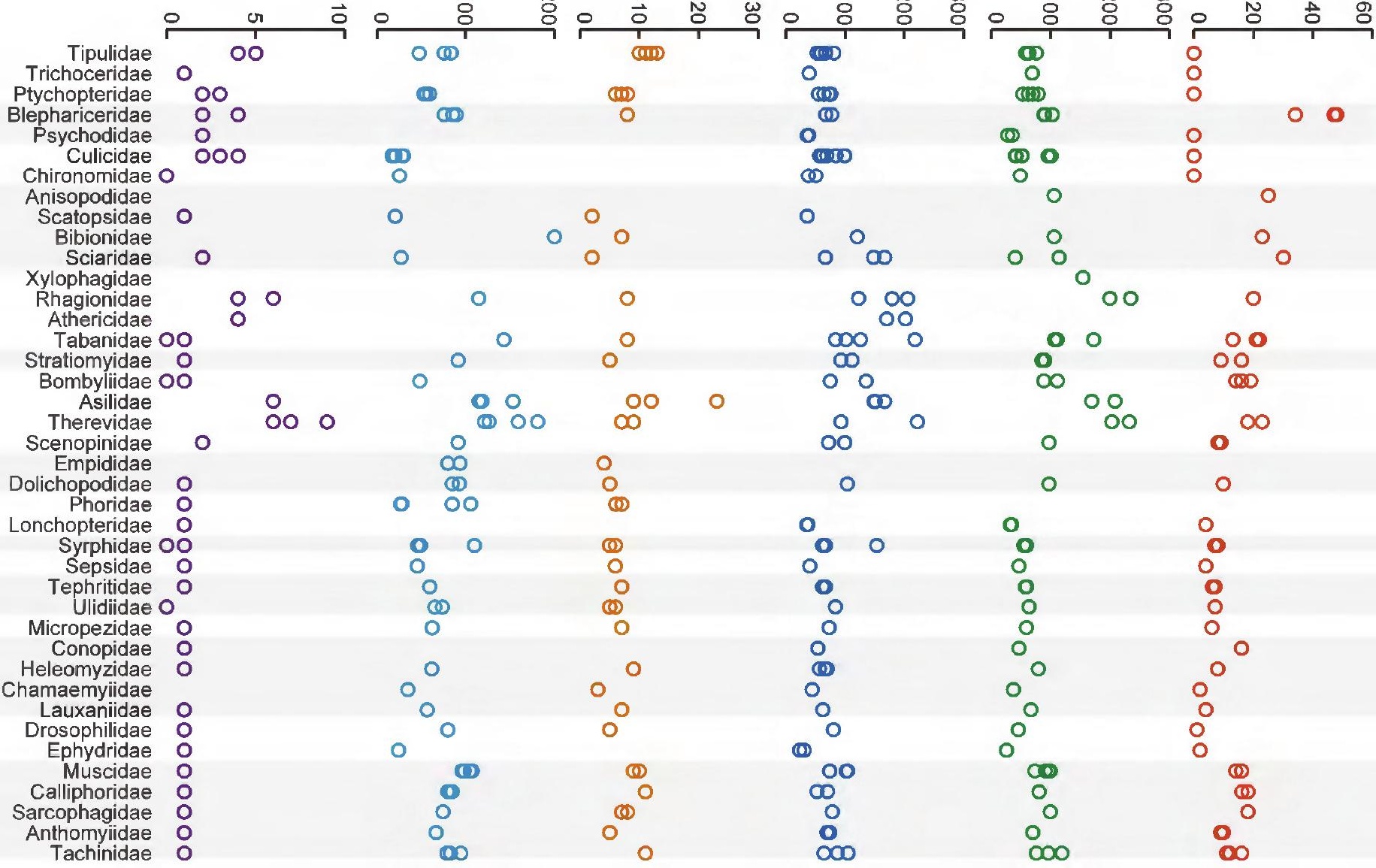


$\begin{array}{cc} & \text { \# of sensilla } \\ \text { vSP } & \mathrm{dSP}\end{array}$

$\infty$ density (sensilla/um²)
dBP

dHP

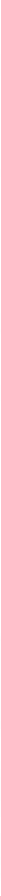






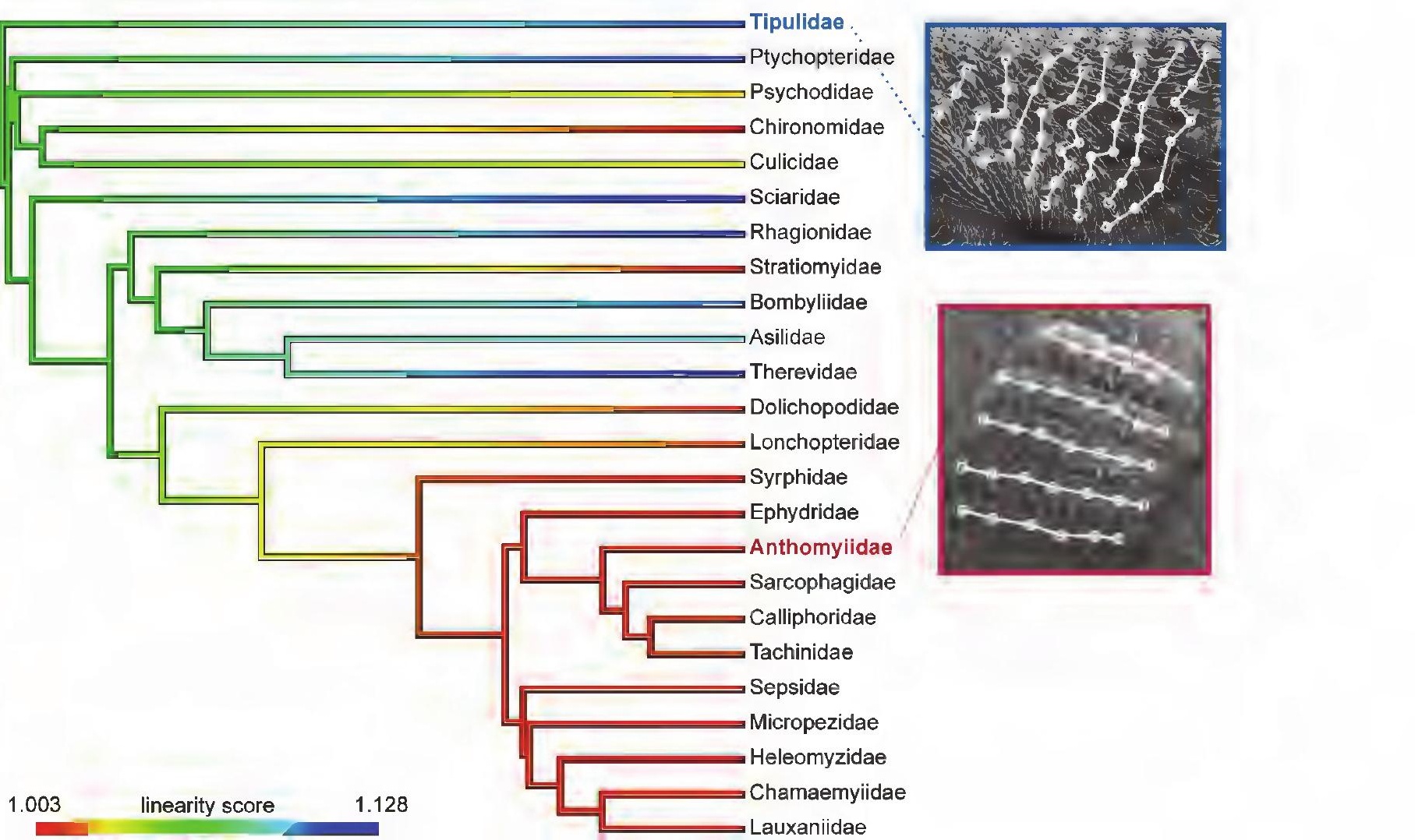



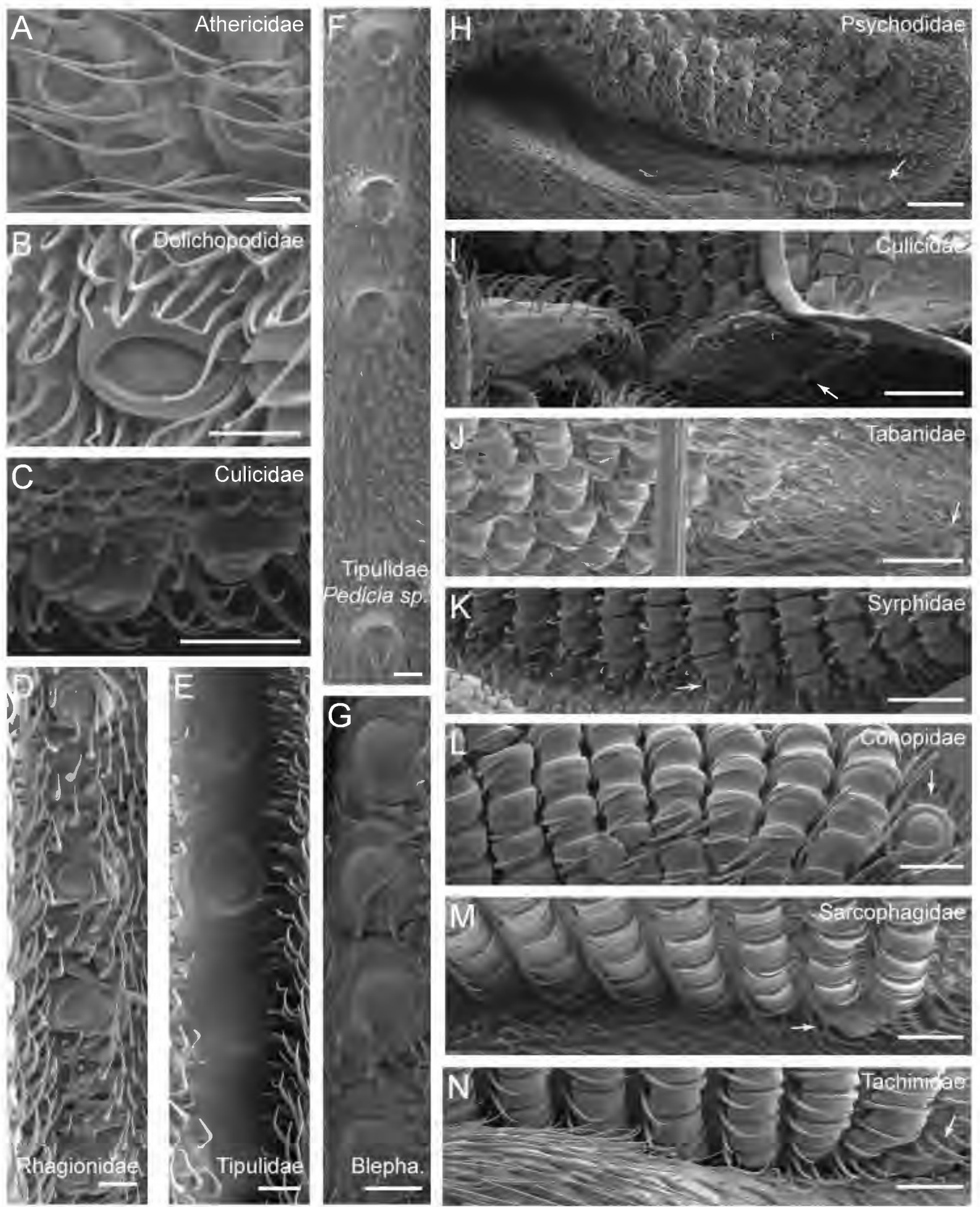

Tipulidae

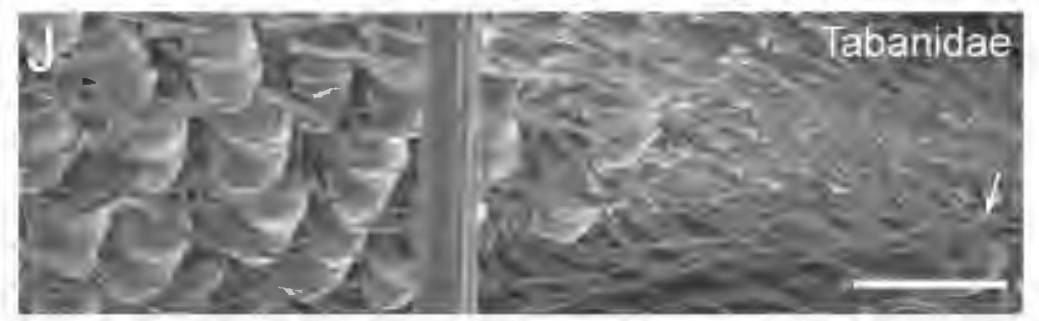

Pedicia sp.
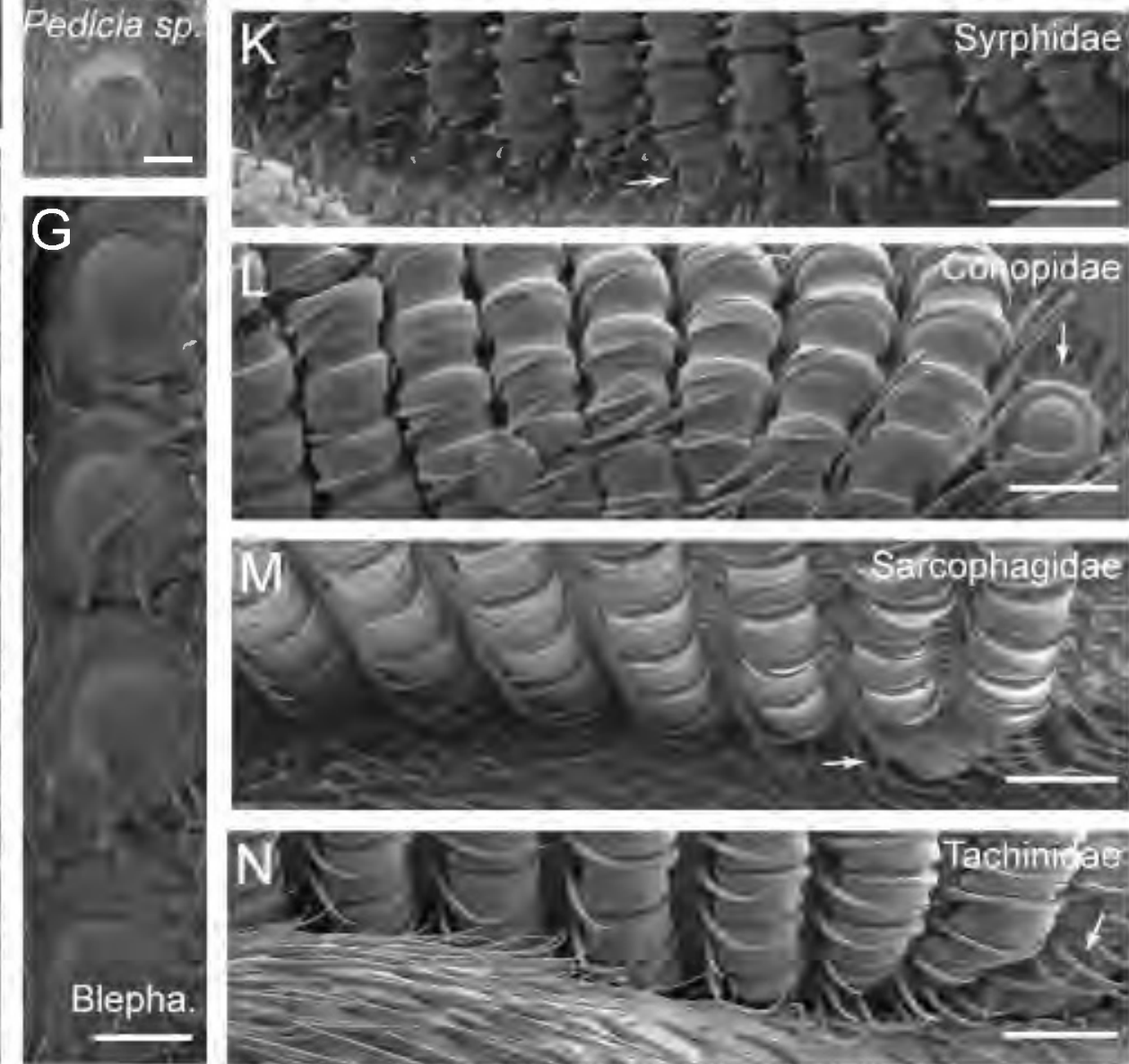

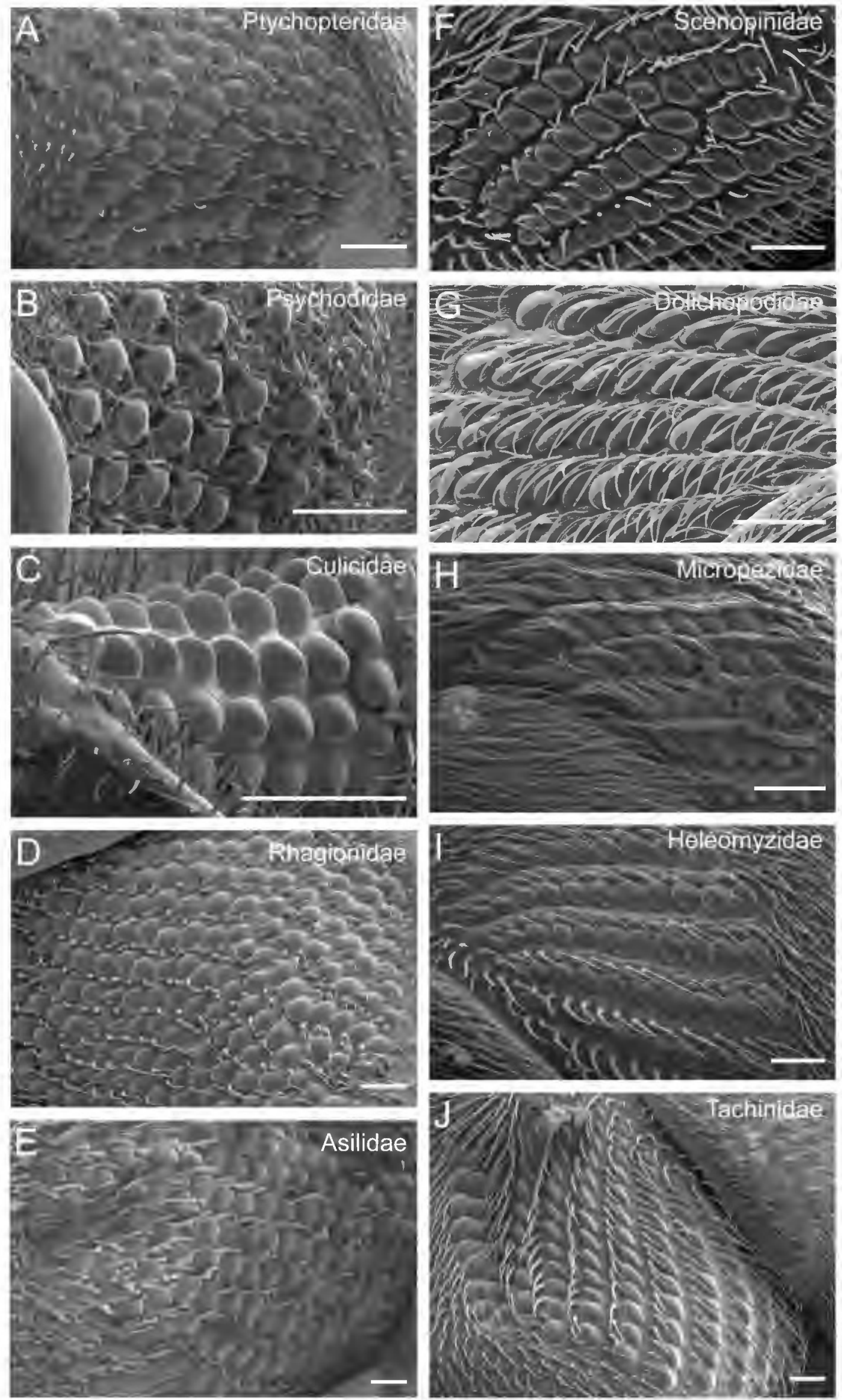


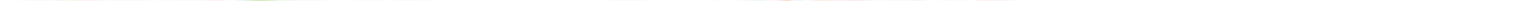



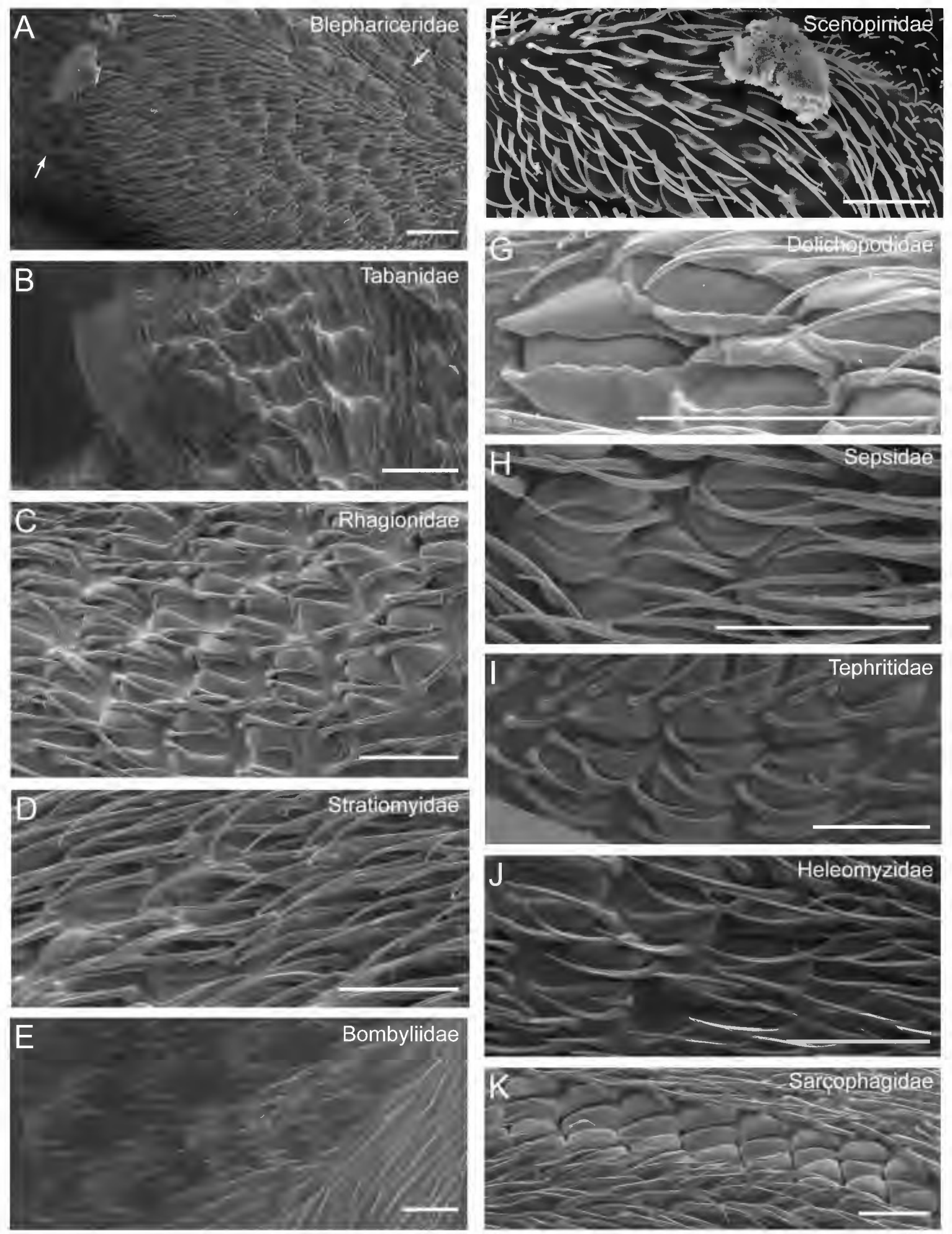